\title{
Hybrid Spintronic Structures With Magnetic Oxides and Heusler Alloys
}

\author{
Xu, Y. B.; Hassan, S. S. A.; Wong, P. K. J.; Wu, Jian; Claydon, J. S.; Lu, Y. X.; Damsgaard, Christian \\ Danvad; Hansen, J. B.; Jacobsen, C. S.; Zhai, Y.
}

Total number of authors:

13

Published in:

I E E E Transactions on Magnetics

Link to article, DOI:

10.1109/TMAG.2008.2002188

Publication date:

2008

Document Version

Publisher's PDF, also known as Version of record

Link back to DTU Orbit

Citation (APA):

Xu, Y. B., Hassan, S. S. A., Wong, P. K. J., Wu, J., Claydon, J. S., Lu, Y. X., Damsgaard, C. D., Hansen, J. B., Jacobsen, C. S., Zhai, Y., van der Laan, G., Feidenhans'I, R. K., \& Holmes, S. N. (2008). Hybrid Spintronic Structures With Magnetic Oxides and Heusler Alloys. I E E E Transactions on Magnetics, 44(11), 2959-2965. https://doi.org/10.1109/TMAG.2008.2002188

\section{General rights}

Copyright and moral rights for the publications made accessible in the public portal are retained by the authors and/or other copyright owners and it is a condition of accessing publications that users recognise and abide by the legal requirements associated with these rights.

- Users may download and print one copy of any publication from the public portal for the purpose of private study or research.

- You may not further distribute the material or use it for any profit-making activity or commercial gain

- You may freely distribute the URL identifying the publication in the public portal 


\title{
Hybrid Spintronic Structures With Magnetic Oxides and Heusler Alloys
}

\author{
Y. B. $\mathrm{Xu}^{1}$, S. S. A. Hassan ${ }^{1}$, P. K. J. Wong ${ }^{1}$, J. Wu${ }^{1}$, J. S. Claydon ${ }^{1}$, Y. X. Lu ${ }^{1}$, C. D. Damsgaard ${ }^{2}$, J. B. Hansen ${ }^{2}$, \\ C. S. Jacobsen ${ }^{2}$, Y. Zhai ${ }^{3}$, G. van der Laan ${ }^{4}$, R. Feidenhans ${ }^{5}$, and S. N. Holmes ${ }^{6}$ \\ ${ }^{1}$ Spintronics and Nanodevice Laboratory, Departments of Electronics and Physics, University of York, York, YO10 5DD, U.K. \\ ${ }^{2}$ Department of Physics and Department of Micro and Nanotechnology, Technical University of Denmark, \\ Lyngby, DK-2800, Denmark \\ ${ }^{3}$ Department of Physics, Southeast University, Nanjing 210096, China \\ ${ }^{4}$ Diamond Laboratory, Oxford OX11 ODE, U.K. \\ ${ }^{5}$ Niels Bohr Institute, Copenhagen University, Copenhagen DK-1168, Denmark \\ ${ }^{6}$ Toshiba Research Europe Ltd., Cambridge Research Laboratory, Cambridge CB4 0WE, U.K.
}

\begin{abstract}
Hybrid spintronic structures, integrating half-metallic magnetic oxides and Heusler alloys with their predicted high spin polarization, are important for the development of second-generation spintronics with high-efficient spin injection. We have synthesized epitaxial magnetic oxide $\mathrm{Fe}_{3} \mathrm{O}_{4}$ on $\mathrm{GaAs}(100)$ and the unit cell of the $\mathrm{Fe}_{3} \mathrm{O}_{4}$ was found to be rotated by $45^{\circ}$ to match the gallium arsenide $\mathrm{GaAs}$. The films were found to have a bulk-like moment down to 3-4 $\mathrm{nm}$ and a low coercivity indicating a high-quality magnetic interface. The magnetization hysteresis loops of the ultrathin films are controlled by uniaxial magnetic anisotropy. The dynamic response of the sample shows a heavily damped precessional response to the applied field pulses. In the Heusler alloy system of Co $\mathrm{MnGa}_{2}$ on GaAs, we found that the magnetic moment was reduced for thicknesses down to $10 \mathrm{~nm}$, which may account for the lower than expected spin-injection efficiency from the spin-light-emitting diode structures. Using the element-specific technique of X-ray magnetic circular dichroism (XMCD), the reduced spin moments were found to originate from the Mn rather than the Co atoms, and the improvement of the interface is thus needed to increase the spin injection efficiency in this system. Further studies of the $I-V \operatorname{characteristics~of~} \mathrm{Fe}_{3} \mathrm{O}_{4} / \mathrm{GaAs}(100)$ and $\mathrm{Fe}_{3} \mathrm{O}_{4} / \mathrm{MgO} / \mathrm{GaAs}(100)$ show that the $\mathrm{Fe}_{3} \mathrm{O}_{4}$-based spintronic structures have a well-defined Schottky or tunneling barrier of moderate barrier height, which is encouraging for high-efficient spin injection.
\end{abstract}

Index Terms-Gallium arsenide (GaAs), Heusler alloys, magnetic oxides, magnetite, molecular-beam epitaxy, spintronics, ultra-thin films.

\section{INTRODUCTION}

$\mathbf{T}$ HE discovery of giant magnetoresistance in the metallic multilayers in the early 1980s initiated and propelled spintronics research, resulting in the first generation of a spintronic device in the form of the spin valve for recording heads. This was followed by the discovery of tunneling magnetoresistance, leading to the magnetic tunnel junction (MTJ) that has been utilized in developing magnetic random-access memory (MRAM). The second-generation spintronics, integrating magnetic materials with semiconductor devices, will not only improve the existing capabilities of electronic devices, but will add new functionalities, enabling future computers to run faster, while consuming less energy, which is expected to have an impact comparable to the development of the transistor 50 years ago. However, magnetic materials and semiconductors have been developed in parallel quite separately over the decades with magnetic materials mainly used for data storage, such as computer hard disks and semiconductor devices for data processing, such as the field-effect transistor (FET) and metal-oxide semiconductor field-effect transistor (MOSFET) in the IT industry. It is thus a major challenge to integrate

Digital Object Identifier 10.1109/TMAG.2008.2002188

Color versions of one or more of the figures in this paper are available online at http://ieeexplore.ieee.org. magnetic materials with semiconductors for the second-generation spintronics [1]. There are currently two classes of material systems exploited: 1) dilute magnetic semiconductors (DMS), such as $\mathrm{Ga}_{1-x} \mathrm{Mn}_{x}$ As and $\mathrm{Cd}_{1-x} \mathrm{Co}_{x} \mathrm{Se}$, and 2) magnetic/semiconductor hybrid spintronic structures (HySpin), such as $\mathrm{Fe} / \mathrm{GaAs}, \mathrm{Co} / \mathrm{Si}, \mathrm{Fe}_{3} \mathrm{O}_{4} / \mathrm{GaAs}, \mathrm{Co}_{2} \mathrm{MnGa} / \mathrm{GaAs}$. DSMs have the great advantage of integrating with conventional semiconductor materials and devices [2]-[4]. However, it remains a real challenge to increase their Curie temperature. The highest $T_{c}$ obtained so far in III-V and II-VI DMSs is $\sim 150 \mathrm{~K}$. To overcome the low solubility of magnetic impurities, such as Mn in semiconductor compounds, DMSs have been grown at a low temperature to avoid secondary-phase formation. Postgrowth annealing has also been adopted to further improve the sample structures and increase the Curie temperature. Some new DMSs have been discovered in the last few years that might - or might not-exhibit ferromagnetic behavior above room temperature, such as Co-doped $\mathrm{TiO}_{2}\left(\mathrm{Co}_{x} \mathrm{Ti}_{1-x} \mathrm{O}_{2}\right)$. On the other hand, hybrid spintronic structures (HySpin) have the advantages of high Curie temperatures, well-controlled magnetic properties, and easy integration with current magnetic technologies. Recently, an encouraging approach to achieve high-efficient spin injection into semiconductors in the diffusive regime has been the use of a spin-dependent energy barrier, such as $\mathrm{MgO}$, which is a good insulating material with high thermal stability. Spin-injection efficiency as high as $55 \%$ at $100 \mathrm{~K}$ has already been demonstrated by Parkin et al. [5] in a $\mathrm{CoFe} / \mathrm{MgO} / \mathrm{GaAs}$ tunneling structure. The ferromagnetic metal/semiconductor hybrid structures [6]-[8] are so far the most extensively 
studied hybrid spintronic materials. The growth of epitaxial ferromagnetic metal/semiconductor hybrid structures was first demonstrated in $\mathrm{Fe} / \mathrm{GaAs}$ by Prinz's group at the Naval Research Laboratory. This is possible, in part, due to the fact that the lattice constant of bcc Fe is approximately half that of GaAs. To achieve high spin injection into semiconductors, the development of high-spin-polarized magnetic materials and their integration with the semiconductor are essential. Half-metallic $\mathrm{Fe}_{3} \mathrm{O}_{4}$ has attracted great attention recently as it has high polarization at the Fermi level and relatively high electrical conductivity at room temperature, which is believed to benefit the injection of spin carriers into the semiconductors. The epitaxial growth of $\mathrm{Fe}_{3} \mathrm{O}_{4}$ on $\mathrm{MgO}$ [9]-[11] $\mathrm{Al}_{2} \mathrm{O}_{3}$ and $\mathrm{Pt}$ [12] has been demonstrated by several groups and several interesting magnetic properties, such as superparamagnetism, slow saturation behavior, and local out-of-plane magnetic moments in zero field, have been observed. In order to incorporate $\mathrm{Fe}_{3} \mathrm{O}_{4}$ into spintronics devices, the synthesis of $\mathrm{Fe}_{3} \mathrm{O}_{4}$ on semiconductor substrates, such as GaAs, is essential. Heusler alloys are considered to be promising materials for spin injectors because they belong to the material class of magnetic half metals. Half metals are magnetic materials with a band gap at the Fermi level for one spin direction and continuous bands for the opposite spin direction. Due to this band asymmetry, the minority electrons are semiconducting and the majority electrons persist their normal metallic characteristics, which consequently gives rise to a situation where the conduction electrons at the Fermi level are 100\% spin polarized [13]. Heusler alloys also possess the properties of having matching lattice constants with III-V semiconductors and high Curie temperatures ranging from 200 to $1200 \mathrm{~K}$.

In this paper, we report our studies of growth, structure analysis, and magnetic properties of hybrid spintronic materials and devices, in particular, with the magnetic oxides and Heusler alloys. All samples were grown by the molecular beam epitaxy (MBE) technique. We focus on two systems: $\mathrm{Fe}_{3} \mathrm{O}_{4}$ and $\mathrm{Co}_{2}$ $\mathrm{MnGa}$, on GaAs-based spintronic structures. The full Heusler alloy $\mathrm{Co}_{2} \mathrm{MnGa}$ is one of the most promising Heusler alloys for the integration with the semiconductor since its lattice matches very well with III-V semiconductor, such as GaAs. We have previously shown that epitaxial $\mathrm{Fe}_{3} \mathrm{O}_{4}$ could be grown on GaAs despite their relatively large lattice mismatch [14]. Our study emphasizes the growth, structures, and interface and magnetic properties since they are critically important to understand and control the spin injection in hybrid spintronics devices.

\section{Epitaxial Growth AND Stuctures}

\section{A. $\mathrm{Fe}_{3} \mathrm{O}_{4} / \mathrm{GaAs}$ and $\mathrm{Fe}_{3} \mathrm{O}_{4} / \mathrm{MgO} / \mathrm{GaAs}$}

There are essentially two different approaches to synthesize $\mathrm{Fe}$ oxides from atomic $\mathrm{Fe}$. The first one is the growth of $\mathrm{Fe}$ in an oxygen environment at a suitable substrate temperature. The second one is first to grow $\mathrm{Fe}$ on the substrate and then to use an oxidant agent to oxidize the $\mathrm{Fe}$ to iron oxide. In the first approach, the incident Fe atoms in the vapor beam are oxidized before reaching the surface and being deposited. This method generates a homogeneous chemical composition throughout the film. However, there are some problems related to this method.
First, the oxidation of the substrate surface (e.g., the GaAs(100) surface) is problematic. In the oxidant agent environment, which should be constructed prior to the Fe oxide growth, the GaAs(100) itself might be oxidized before Fe oxide deposition. This incurs a GaAs(100) oxide interface between the bulk GaAs and the Fe oxide. Second, there is a possible chemical stoichiometric variation depending on the oxidant agent and substrate temperature, etc. Third, the growth of Fe oxide on the Fe oxide might induce antiphase boundaries (APBs) in the films. The second approach is postgrowth oxidation, where $\mathrm{Fe}$ is epitaxially grown on the $\mathrm{GaAs}(100)$ prior to oxidation. This approach leads to an ultrathin Fe oxide film of high quality owing to the oxidation mechanism applied here. It is known that in the oxidation process of thick Fe film, the electrons migrate from the inner atomic Fe/oxide interface to the oxide/oxygen surface and combine with oxygen to form $\mathrm{O}^{2-}$ anions. The $\mathrm{Fe}$ oxides, going from $\mathrm{Fe}$ to oxygen rich, range from $\mathrm{FeO}$, $\mathrm{Fe}_{3} \mathrm{O}_{4}$ to $\mathrm{Fe}_{2} \mathrm{O}_{3}$. Due to the intrinsic nature of the oxide, the thickness ratio of each oxide depends on the potential energy. If the Fe film is ultrathin, such as a few nanometers, then the composition dependence on depth might disappear and uniform stoichiometry might be achieved.

We have developed this second approach to grow the $\mathrm{Fe}_{3} \mathrm{O}_{4}$ films on GaAs. Ultrathin single crystal Fe films were first grown on the GaAs substrate by MBE, and the sample was exposed to $\mathrm{O}_{2}$ in the growth chamber, maintaining the $\mathrm{O}_{2}$ partial pressure at $5 \times 10^{-5}$ mbar using a leak valve. Fig. 1(a) and (b) shows the RHEED patterns of the $\mathrm{GaAs}(100)$ substrates and a 3-nm-thick $\mathrm{Fe}_{3} \mathrm{O}_{4}$ film after 3 min. of oxidization at a substrate temperature of $500 \mathrm{~K}$. Similar to the discussion of $\mathrm{Fe}$ on the GaAs(100) substrate, the oxide exhibits a fourfold symmetry in the film plane, which gives rise to similar RHEED patterns with the electron beams along the [011] and [0-11] directions. The sharper RHEED pattern in Fig. 1(b) confirms a more uniform $\mathrm{Fe}_{3} \mathrm{O}_{4}$ growth here in comparison with our previous studies [14], which may be due to the well-ordered GaAs surface obtained after in-situ annealing as shown by the clear Kochiki lines. The RHEED patterns also suggested a rotation of $45^{\circ}$ in the growth relationship, namely $\mathrm{Fe}_{3} \mathrm{O}_{4}(100)<011>/ / \mathrm{GaAs}(100)<010>$ with the unit cell of $\mathrm{Fe}_{3} \mathrm{O}_{4}$ rotated by $45^{\circ}$ to match that of the $\mathrm{GaAs}(100)$ substrate. With a $3-\mathrm{nm} \mathrm{MgO}$ interlayer grown at a GaAs substrate temperature of $673 \mathrm{~K}$, an epitaxial relationship, $\mathrm{MgO}(100)<001>/ / \operatorname{GaAs}(100)<001>$ was obtained, as shown by the RHEED pattern of the $\mathrm{MgO}$ included in Fig. 1(c), despite the large lattice mismatch $(25.5 \%)$ of $\mathrm{MgO}$ and GaAs. Further growth of 3-nm film of $\mathrm{Fe}_{3} \mathrm{O}_{4}$ on top of the $\mathrm{MgO} / \mathrm{GaAs}$ structure using the same conditions as described before gives rise to the overall growth relationship of $\mathrm{Fe}_{3} \mathrm{O}_{4}$ $(100)<001>/ / \mathrm{MgO}(100)<001>/ / \operatorname{GaAs}(100)<001>$, which can be seen in Fig. 1(d). Notice that the $\mathrm{Fe}_{3} \mathrm{O}_{4}$ unit cell rotates $45^{\circ}$ back compared to the case of $\mathrm{Fe}_{3} \mathrm{O}_{4} / \mathrm{GaAs}$ because the rotation gives a nearly perfect lattice match between $\mathrm{Fe}_{3} \mathrm{O}_{4}$ and $\mathrm{MgO}$. Using XPS measurements, we have further confirmed that the samples are $\mathrm{Fe}_{3} \mathrm{O}_{4}$ rather than $\gamma-\mathrm{Fe}_{2} \mathrm{O}_{3}$, which has a similar crystal structure as $\mathrm{Fe}_{3} \mathrm{O}_{4}$. The magnetic coupling of the samples was characterized using X-ray magnetic circular dichroism (XMCD), which is capable of probing the magnetic 




Fig. 1. RHEED images of (a) $\mathrm{GaAs}(100)$ substrate. (b) $\mathrm{Fe}_{3} \mathrm{O}_{4}(3 \mathrm{~nm}) / \mathrm{GaAs}$. (c) $\mathrm{MgO} / \mathrm{GaAs}(100)$. (d) $\mathrm{Fe}_{3} \mathrm{O}_{4}(3 \mathrm{~nm}) / \mathrm{MgO} / \mathrm{GaAs}(100)$.

signal from sites of different chemical environments. The ferromagnetic magnetite features an inverse spinel structure in which the tetrahedral sites are entirely occupied by $\mathrm{Fe}_{\mathrm{td}}^{3+}$ cations but the octahedral sites are equally filled by $\mathrm{Fe}_{\mathrm{oh}}^{2+}$ and $\mathrm{Fe}_{\mathrm{oh}}^{3+}$ cations, which has been confirmed by our XMCD measurements of the ultrathin $\mathrm{Fe}_{3} \mathrm{O}_{4}$ films.

\section{B. Heusler Alloys $\mathrm{Co}_{2} \mathrm{MnGa}$ on GaAs and Spin-LED}

Thin films of the ferromagnetic Heusler alloy $\mathrm{Co}_{2} \mathrm{MnGa}$ were grown epitaxially using MBE on $\mathrm{GaAs}(100)$ substrates that were overgrown with $1 \mu \mathrm{m}$ of GaAs and capped with a few $\mu \mathrm{m}$ of amorphous As. Prior to the growth of the Heusler thin films, the surface of the substrates was cleaned in-situ by As desorption in an ultra-high vacuum up to a temperature of $\sim 450{ }^{\circ} \mathrm{C}$. Two effusion cells and an e-gun source provided atomic beams of $\mathrm{Mn}, \mathrm{Ga}$, and $\mathrm{Co}$. Deposition rates were monitored using quartz-crystal microbalances and a quadrupole mass spectrometer. The growth temperature used was $250^{\circ} \mathrm{C}$, which has been chosen for two reasons. First, at lower temperatures, the thin film could have many structural defects, such as dislocations and antisites, and second, but at higher temperatures, where the crystal has only few defects, the diffusion at the interface between substrate and film will form a magnetic dead layer, which could cause spin scattering and quenching of the spin polarization, leading to an unusable material for spintronics device applications. The used growth temperature is thought to be the highest temperature without any confirmed interface diffusion layer and it is similar to that reported to grow some other Heusler alloys $\left(200^{\circ} \mathrm{C}-300^{\circ} \mathrm{C}\right.$ ), such as $\mathrm{NiMnSb}$ [15], [16], $\mathrm{Ni}_{2} \mathrm{MnGa}$ [17], $\mathrm{Co}_{2} \mathrm{MnGe}$ [18] and $\mathrm{Co}_{2} \mathrm{MnSi}$ [19], [20]. Using a mechanical shutter very close to sample during growth, the $\mathrm{Co}_{2} \mathrm{MnGa}$ thin films were fabricated as a staircase comprised of different thicknesses. The $\mathrm{Co}_{2} \mathrm{MnGa}$ layers were finally capped with a $2.0 \mathrm{~nm}$ layer of either $\mathrm{Al}$ or Au. By observing in-situ reflection high-energy electron diffraction (RHEED) patterns during growth, an initial determination of crystal quality was obtained. Fig. 2 shows RHEED patterns taken after the MBE growth of the $\mathrm{Co}_{2} \mathrm{MnGa}$ film on a GaAs p-i-n spin-LED structure.

A high-resolution X-ray diffraction (XRD) technique for $\theta-2 \theta$ scans and $\omega$-scans was used to investigate the detailed crystal structure of the Heusler alloys on GaAs. Fig. 3 illustrates the epitaxial growth of a 9.7-nm $\mathrm{Co}_{2} \mathrm{MnGa}$ film on $\mathrm{GaAs}(100)$.

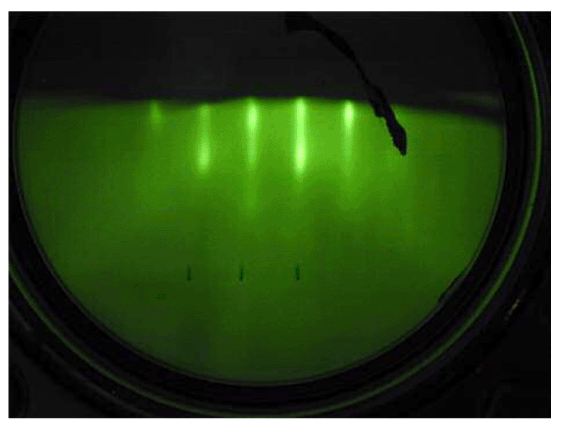

Fig. 2. RHEED (20-kV) patterns after growth of the $\mathrm{Co}_{2} \mathrm{MnGa}$ on the GaAs spin-LED structure.

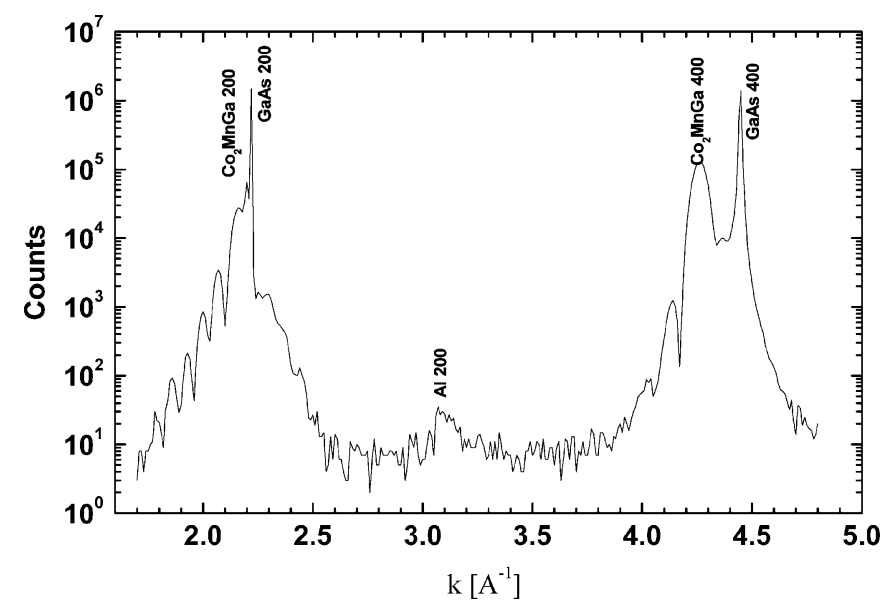

Fig. 3. Synchrotron XRD measurements of 9.7-nm $\mathrm{Co}_{2} \mathrm{MnGa}$ on $\mathrm{GaAs}(100)$.

We found that the film evolves from a strained film with inplane lattice parameters matched with GaAs $a=5.65 \AA$ into a relaxed film with bulk lattice constant $a=5.77 \AA$ at a critical thickness of $\sim 10 \mathrm{~nm}$.

\section{Magnetic Moments of Ultrathin $\mathrm{FE}_{3} \mathrm{O}_{4}$ Films}

$\mathrm{Fe}_{3} \mathrm{O}_{4}$ has a spinel structure, where the unit cell contains two $\mathrm{Fe}^{3+}$ ions equally occupying the tetragonal A site and octahedral $\mathrm{B}$ sites and one $\mathrm{Fe}^{2+}$ ion in the $\mathrm{B}$ site. The local magnetic moments on the $\mathrm{B}$ sites are antiparallel to that on the A site. Thus, the net magnetic moment is contributed solely by the $\mathrm{Fe}^{2+}$ ion, which has an atomic value of $\sim 4 \mu_{B}$, disregarding the contribution of the orbital moment. The total magnetization was measured with vibrating sample magnetometer (VSM) at room temperature. Based on the measured thickness and the area of the Fe films before oxidation, we calculated the number of $\mathrm{Fe}$ atoms in each film. Then, dividing the measured magnetic moment of the $\mathrm{Fe}_{3} \mathrm{O}_{4}$ film by three of the calculated total number of $\mathrm{Fe}$ atoms, we obtain the average numbers of Bohr magneton per formula unit (f.u) in the $\mathrm{Fe}_{3} \mathrm{O}_{4}$ film.

Fig. 4 shows the thickness dependence of the magnetic moment per formula unit of the magnetite films with the nominal thicknesses varying from 2 to $8 \mathrm{~nm}$. A comparison with the theoretical value of $\sim 4 \mu_{B}$ for the magnetic moment of $\mathrm{Fe}_{3} \mathrm{O}_{4}$ shows that for the thinnest film of $2 \mathrm{~nm}$, the moment is less than the theoretical value, which may be understood as being due to an incomplete film structure, such as in epitaxial superparamagnetic-clustered Fe [18], [21]. For the 8-nm film, the magnetic 


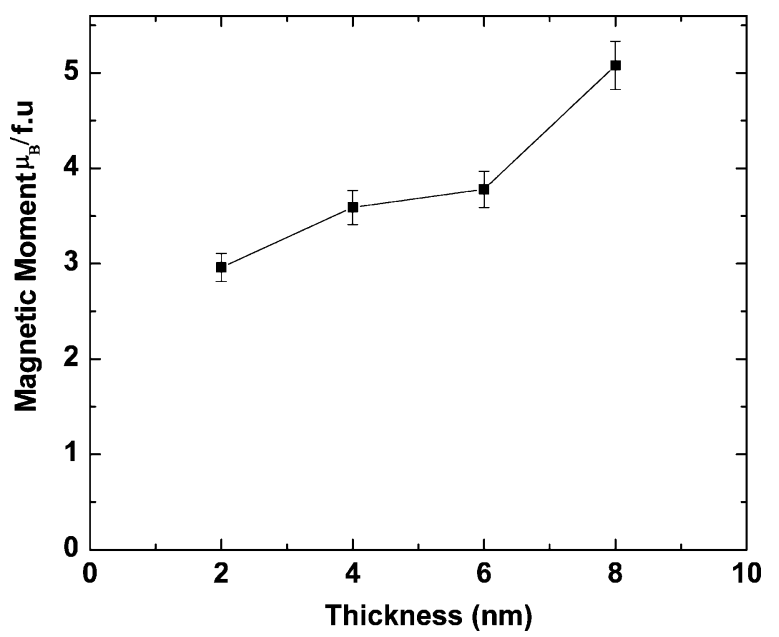

Fig. 4. Thickness dependence of the magnetic moments per formula unit for $\mathrm{Fe}_{3} \mathrm{O}_{4}$ films grown on $\mathrm{GaAs}(100)$.

moment per formula unit is much higher than $4 \mu_{B}$ which might be possibly due to some residual $\mathrm{Fe}$ not being fully oxidized under the $\mathrm{Fe}_{3} \mathrm{O}_{4}$ layer. The magnetization of the 4- and 6-nm films are nearly but slightly less than $4 \mu_{B}$, which suggests that these films are fully oxidized to half metallic $\mathrm{Fe}_{3} \mathrm{O}_{4}$ and have almost stoichiometric composition. We also like to point out that the magnetic moment of the $\mathrm{Fe}_{3} \mathrm{O}_{4}$ films might contain a contribution from the orbital magnetic moment, which requires further study.

\section{INTERFACE MAGNETIZATION AND SPIN-INJECTION EFFICIENCY WITH $\mathrm{CO}_{2}$ MNGA}

Most of the reported magnetization measurements of Heusler alloys have been on bulk materials or thick films. We have recently studied the ultrathin Heusler films of less than $10 \mathrm{~nm}$ with XMCD and VSM measurements in order to obtain some insight in the interface magnetic properties, which is critical to spin-injection in various spin devices such as spin-LED. Mn and Co $L_{2,3} \mathrm{X}$-ray absorption (XA) and XMCD spectra were recorded at room temperature using $70 \%$ circularly polarized soft X-rays from beamline 1.1 at the Synchrotron Radiation Source (SRS) in Daresbury Laboratory, UK. Magnetic fields up to $0.6 \mathrm{~T}$ were applied parallel and antiparallel to the photon helicity vector using the Portable Octupole Magnet System (POMS). XA spectra were collected using the total-electron yield (TEY) detection method with the sample surface inclined at an angle of $30^{\circ}$ to the X-ray beam. Fixed photon helicity was used to obtain the XMCD signal at each point of the spectrum by reversing the direction of the applied magnetic field along the X-ray beam. A typical set of XMCD spectra for both the $\mathrm{Mn}$ and $\mathrm{Co}$ edges from $9.7 \mathrm{~nm} \mathrm{\textrm {Co } _ { 2 }} \mathrm{MnGa}$ on $\mathrm{GaAs}(100)$ is shown in Fig. 5. The spectra were analysed quantitatively by applying the sum rules to assess the element-specific magnetic moments [21], [22] and the results are summarised in Table I. The difference between the magnetic moment values from the VSM and XMCD measurements could be associated with the physical limitations on probing depth using the XMCD technique since soft $\mathrm{X}$-rays are expected to penetrate only to depths of up to $5 \mathrm{~nm}$ [23]. Both the XMCD and VSM measurements

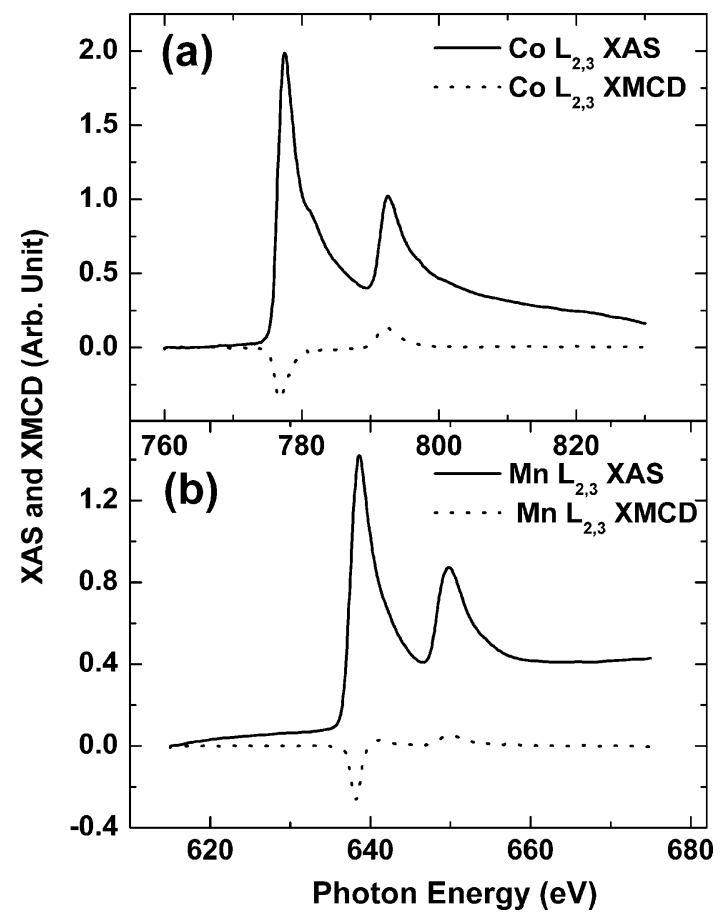

Fig. 5. XA and XMCD spectra for $9.7 \mathrm{~nm} \mathrm{Co} 2 \mathrm{MnGa} / \mathrm{GaAs}(100)$ samples. (a) Co $L_{2,3}$ and (b) $\operatorname{Mn} L_{2,3}$ edge.

suggest that the magnetic moments are greatly reduced in the ultrathin films and, thus, at the interface. The Co sites still have a moment close to the theoretical values, but a large transfer of the magnetic moment from the Co to the Mn sites appears to be absent despite the average Co moment being reduced from its bulk value of $\sim 1.7 \mu_{B}$ /atom [24]. The reason behind these discrepancies is not yet fully clear. Though the tiny shoulders of the Co L2 and L3 edges might indicate a very slight oxidization of the Co, it is, however, unlikely to cause the reduced moments at the Mn site. Calculations carried out by the group from DTU and $\mathrm{KU}$ have suggested that Mn defects residing on Co sites may antialign themselves with the applied field [25]. Based on depth profiling Auger data [24], we have found that Mn tends to diffuse toward the surface into Al. The large change of the magnetic moment upon increasing the thickness from 5.3 to 7.6 $\mathrm{nm}$ suggests that there might be an interface layer with a much lower magnetic moment than the rest of the film. Its origin appears to be due to the diffusion of substrate atoms into the alloy films, which is known to significantly change the local magnetic moments [26], [27].

We have carried out optical measurements in the oblique Hanle effect geometry to measure the electrical spin injection into an InGaAs quantum well in the i-region of a GaAs p-i-n LED device with Heusler alloy spin injectors. The first $\mathrm{Co}_{2}$ MnGa Heusler alloy spin LED [29] was fabricated on a highly doped $\mathrm{Al}_{0.3} \mathrm{Ga}_{0.7}$ As Schottky barrier that had already been optimized for spin injection with Fe. Hanle geometry electroluminescence measurements determined that the spin injection from the nonstoichiometric alloy $\mathrm{Co}_{2.4} \mathrm{Mn}_{1.6} \mathrm{Ga}$ was $13 \%$ efficient at $5 \mathrm{~K}$, which meant the optical polarization efficiency in the spin LED was only $2.4 \%$. An interfacial disorder limited this value and in addition, the polarization signal disappeared 
TABLE I

XMCD Results For the LoCAl MaGNETIC MOMENTS OF Co AND MN, COMPARED WITH THE VSM SATURATION MAGNETIZATION MEASUREMENT FOR $\mathrm{CO}_{2} \mathrm{MnGA}$. The MEASurements ARe Performed at $300 \mathrm{~K}$. $\mathrm{m}_{\text {bulk }}=3.71 \mu \mathrm{B}$ HAS BEEN CHOSEN TO BE $90 \%$ OF THE THEORETICALLY EXPECTED LOW TEMPERATURE VALUE [25]. $\mathrm{M}_{\text {Bulk }}$ Is TAKEN To BE 711 EMU/CM ${ }^{3}$, WEBSTER [28]

\begin{tabular}{|c||c|c|c|c||}
\hline $\begin{array}{c}\text { Thickness } \\
(\mathbf{n m})\end{array}$ & $\begin{array}{c}\text { Mn } \mathbf{m}_{\text {total }} \\
\left(\boldsymbol{\mu}_{\mathbf{B}}\right)\end{array}$ & $\begin{array}{c}\mathbf{C o} \mathbf{m}_{\text {total }} \\
\left(\mu_{\mathrm{B}}\right)\end{array}$ & $\begin{array}{c}\mathbf{X M C D} \\
\left(\mathbf{m}_{\mathbf{M n}}+\mathbf{2} \mathbf{m}_{\mathbf{C}}\right) / \mathbf{m}_{\text {bulk }}\end{array}$ & $\begin{array}{c}\mathbf{V S M} \\
\mathbf{C o}_{\mathbf{2}} \mathbf{M n G a} / \mathbf{M}_{\text {bulk }}\end{array}$ \\
\hline \hline 5.3 & 0.534 & 0.175 & 0.19 & 0.15 \\
\hline 7.6 & 0.724 & 0.305 & 0.46 & 0.61 \\
\hline 9.7 & 0.470 & 0.341 & 0.23 & 0.70 \\
\hline Bulk [28] & 3.01 & 0.52 & & \\
\hline
\end{tabular}

above $20 \mathrm{~K}$. The same spin LED with an Fe contact could operate at $300 \mathrm{~K}$ with $1 \%$ efficiency and at $5 \mathrm{~K}$ with $31 \%$ efficiency. At low temperatures, a dynamic nuclear polarization signal could be observed with the Heusler alloy contact, which meant that subtle spin interaction effects could still be observed in the semiconductor device even with relatively inefficient spin injection. $\mathrm{A} \mathrm{Co}_{2} \mathrm{MnGe}$ Heusler alloy spin LED [30] had an efficiency of $27 \%$, which represents the limiting value with an epitaxial Heusler alloy even when the band structure is predicted to be half metallic. In more recent stoichiometric $\mathrm{Co}_{2} \mathrm{MnGa}$ structures [31], the spin-injection efficiency is $22 \%$. This lower than expected spin-injection efficiency is correlated with the reduced interface magnetization as observed from the XMCD and VSM measurements.

\section{Static AND Dynamic SWITCHING}

As a bulk ferromagnetic ferrite, magnetite shows a cubic magnetic anisotropy, with the easy, medium, and hard axes along the $<111>,<110>$, and $<100>$ directions, respectively, above $130 \mathrm{~K}$. Using MOKE measurements, we have studied the magnetization hysteresis loops along four major directions. We found that the films show strong uniaxial magnetic anisotropy in a thickness range of $2-8 \mathrm{~nm}$, as illustrated in Fig. 6 by the MOKE loops from a 3-nm film. The easy axis of the uniaxial anisotropy is along the [0-11] orientation of the GaAs $(100)$ substrate. The films have a relatively low coercivity of only $12 \mathrm{Oe}$ as expected from epitaxial growth. The saturation field along the hard axis is $80 \mathrm{Oe}$, which is proportional to the uniaxial magnetic anisotropy fields. The influence of cubic anisotropy $\left(K_{1}\right)$ becomes stronger compared to that of the uniaxial $\left(K_{u}\right)$ with increasing thickness. As a consequence of the increase in the ratio of $K_{1} / K_{u}$, the global easy axis rotates from the uniaxial easy [0-11] direction to the cubic easy [001] direction, when the thickness is above $6 \mathrm{~nm}$. The uniaxial magnetic anisotropy might be related to the interface as that observed in the $\mathrm{Fe} / \mathrm{GaAs}$ system. There are generally two mechanisms associated with "unidirectional chemical bonding" and "magnetoelastic coupling," respectively, that explain the uniaxial magnetic anisotropy in the magnetic/semiconductor hybrid structures. These two mechanisms might be responsible for the uniaxial magnetic anisotropy observed here. However, the interface of $\mathrm{Fe}_{3} \mathrm{O}_{4} / \mathrm{GaAs}(100)$ is even more complicated than that of $\mathrm{Fe} / \mathrm{GaAs}(100)$ as first the ultrathin $\mathrm{Fe}_{3} \mathrm{O}_{4}$ itself might be different from the bulk, and second, the bonding of oxygen to the semiconductor surface is not clear so far.

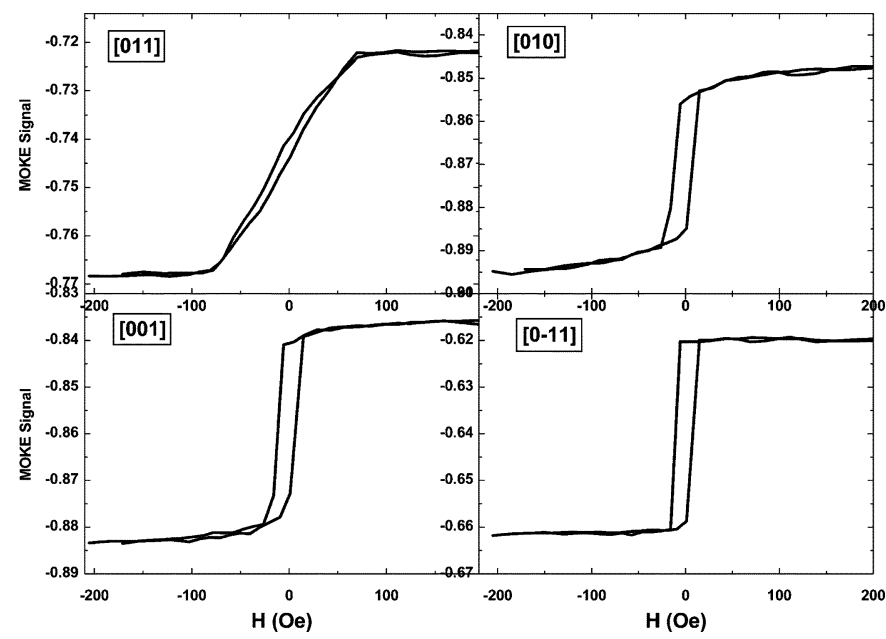

Fig. 6. MOKE loops from 3-nm $\mathrm{Fe}_{3} \mathrm{O}_{4} / \mathrm{GaAs}(100)$ along: (a) [011]. (b) [001]. (c) $[0-11]$. (d) [010].

Dynamic switching has also been measured in the single-crystal $\mathrm{Fe}_{3} \mathrm{O}_{4}$ using MOKE techniques. The picosecond magnetization dynamics induced by a pulsed magnetic field have a precessional character that is sensitive to the temporal profile and orientation of the pulsed field and the magnetic properties of materials, such as magnetic anisotropies and magnetic damping that are present. Dynamic magnetization switching measurements provide essential information for the application of this material system into spintronic devices, such as understanding the ultimate limit of the switching speed [32], [33]. A field-pumped time-resolved magneto-optical Kerr effect (TRMOKE) setup has been used to investigate time-resolved magnetization dynamics of epitaxial $\mathrm{Fe}_{3} \mathrm{O}_{4}$ ultra-thin films [34]. The field pulse used to trigger the dynamics has a 10-90\% rise time of $350 \mathrm{ps}$, a decay time of $\sim 1 \mathrm{~ns}$, and a peak field of $1 \mathrm{kOe}$. The magnetization dynamics of the sample are probed using the polar MOKE effect, giving a measurement of the out-of-plane component of magnetization. The dynamic response of the sample to the field pulse was measured for different dc bias fields applied along two perpendicular crystalline directions. Fig. 7 shows the measured dynamic response of the 3-nm sample to the field pulse at a dc bias field of 720 Oe. By fitting the Landau-Lifshitz-Gilbert (LLG) equation, we found that the damping constant of the $\mathrm{Fe}_{3} \mathrm{O}_{4}$ ultrathin films is $\sim 0.1$, significantly exceeding that of its metallic Fe counterpart of $\sim 0.01$. This might be related to the ferromagnetic coupling in $\mathrm{Fe}_{3} \mathrm{O}_{4}$ as well as the interface bonding and magnetoelastic coupling as indicated by the static hysteresis measurements. The heavy damping suggests a potentially higher working frequency of spintronic devices made of this half metallic material.

\section{INTERFACE SCHOTTKY BARRIER AND TUNNELING BARRIER}

Apart from the fundamental magnetic properties, the nature of the interface contact and the barrier height is also one of the key issues for the hybrid spintronic devices. We have carried out $I-V$ measurements across the $\mathrm{Fe}_{3} \mathrm{O}_{4}$ and n-doped GaAs interface and the results are shown in Fig. 8(a). The nonlinear behavior in the low-bias region can be attributed to the Schottky 


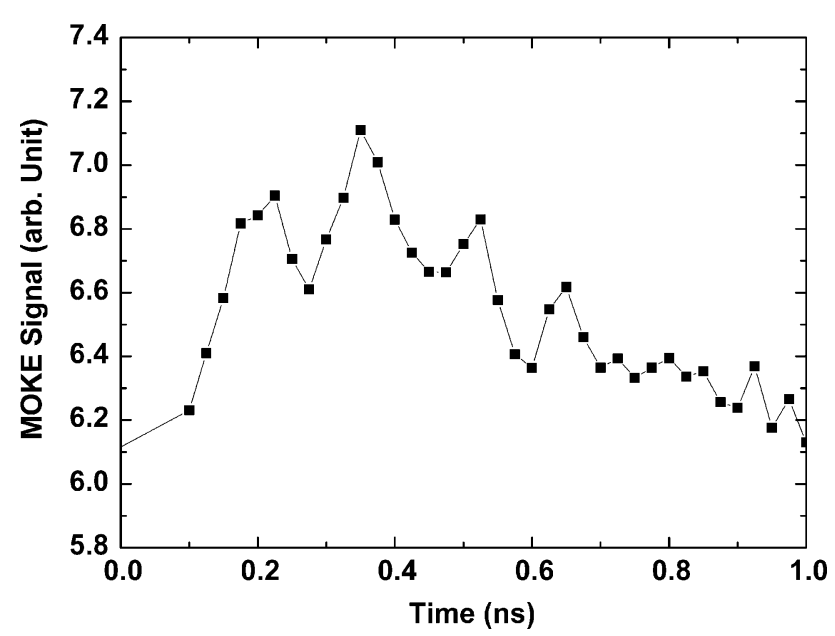

Fig. 7. Time-domain TRMOKE measurements of the 3-nm sample along the [011] direction.

barrier near the interface. The forward and reverse current resulted in an asymmetric bias voltage. This diode-like behavior is typical for Schottky barriers. There are three different mechanisms responsible for the Schottky effect: 1) diffusion of the carrier from the semiconductor to the magnetite thin film; 2) thermionic emission of the carrier across the Schottky barrier; or 3) quantum mechanical tunnelling through the barrier. We found that the thermionic emission-diffusion model best fits the experimental results. Assuming an effective Richardson constant [35] of $8.2 \mathrm{~A} \mathrm{~cm}^{-2} \mathrm{~K}^{-2}$, the Schottky barrier height was found to be $0.34 \mathrm{eV}$. This is about half of the Schottky barrier height of the metal/GaAs contact, such as $\mathrm{Fe} / \mathrm{GaAs}$, which lies within the range $0.69-0.72 \mathrm{eV}$. It is interesting to note that the Schottky barrier height of the MBE-grown magnetite is less than the $0.58-0.63 \mathrm{eV}$ Schottky barrier height observed by Watts et al. [36] in the magnetite thin films deposited on top of the $\operatorname{GaAs}(100)$ by reactive dc sputtering. This indicates that the Schottky barrier height of the $\mathrm{Fe}_{3} \mathrm{O}_{4} / \mathrm{GaAs}$ contact depends on the growth condition, which may be controlled to have the desired barrier heights for spin injection.

We have further characterized the $\mathrm{Fe}_{3} \mathrm{O}_{4}$-based structures with the $\mathrm{MgO}$ interface and the result is included in Fig. 8(b). It is interesting to observe that the $I-V$ curve is much less asymmetric than that without the $\mathrm{MgO}$ barrier. The result suggests that electrical current in the $\mathrm{Fe}_{3} \mathrm{O}_{4}(4 \mathrm{~nm}) / \mathrm{MgO} / \mathrm{GaAs}(100)$ structure is determined by the tunneling process between the magnetite and GaAs through the $\mathrm{MgO}$ barrier. We have fitted the $I-V$ curve with the Simmons model [37] which is capable of determining the barrier width and height of the tunnelling structure. We found that the barrier width and height obtained from the best fit of the Simmons model are $3 \mathrm{~nm}$ and $0.8 \mathrm{eV}$, respectively, for the $\mathrm{Fe}_{3} \mathrm{O}_{4} / \mathrm{MgO} / \mathrm{n}-\mathrm{GaAs}$ junctions. Detailed simulations will be published elsewhere. The fitted barrier height is comparable to the values reported for MTJs with $\mathrm{MgO}$ barriers deposited by various techniques [38], [39], whereas the barrier width is in good agreement with the calibrated thickness of the $\mathrm{MgO}$. The full epitaxial $\mathrm{Fe}_{3} \mathrm{O}_{4} / \mathrm{MgO} / \mathrm{GaAs}(100)$ structure combining the half metallic $\mathrm{Fe}_{3} \mathrm{O}_{4}$ and high-quality

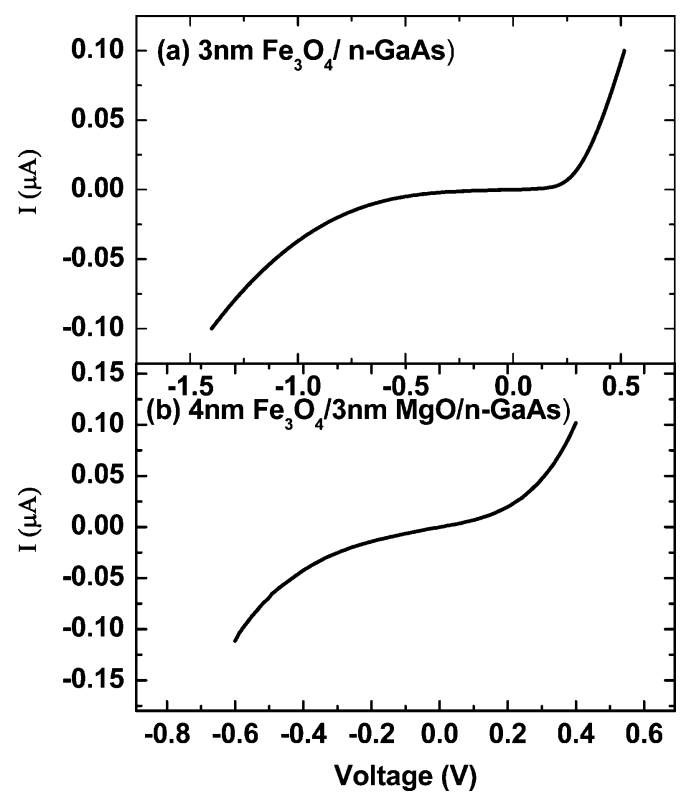

Fig. 8. $I-V$ curves from (a) $\mathrm{Fe}_{3} \mathrm{O}_{4}(3 \mathrm{~nm}) / \mathrm{GaAs}(100)$ and (b) $\mathrm{Fe}_{3} \mathrm{O}_{4}(4 \mathrm{~nm}) /$ $\mathrm{MgO} / \mathrm{GaAs}(100)$.

$\mathrm{MgO}$ barrier forms a promising platform of high-efficient spin injection for spintronic applications.

\section{CONCLUSION}

We have reported the growth, structure, interface, and magnetic properties of magnetic/semiconductor hybrid spintronic structures based on half-metallic oxides and Heusler alloys. By using postgrowth annealing, we have synthesized the epitaxial magnetic oxide $\mathrm{Fe}_{3} \mathrm{O}_{4}$ on $\mathrm{GaAs}(100)$ and the unit cell of the $\mathrm{Fe}_{3} \mathrm{O}_{4}$ was found to rotate by $45^{\circ}$ to match GaAs. The films were found to have a bulk-like moment down to 3-4 nm, indicating a magnetic interface suitable for high-efficient spin injection. The ultrathin films have a low coercivity of around 12Oe, and the magnetization is controlled by a uniaxial anisotropy related to the strain or chemical bonding at the interface. The dynamic measurements show that the films have heavy damping and, thus, can be switched fast. A moderate Schottky barrier of around $0.3 \mathrm{eV}$ was found at the $\mathrm{Fe}_{3} \mathrm{O}_{4} / \mathrm{GaAs}$ interface, which is favorable for spin injection. In the Heusler alloy systems, such as $\mathrm{Co}_{2} \mathrm{MnGa}$ on GaAs, we found the magnetic moment was reduced by more than $50 \%$ for the thickness down to $10 \mathrm{~nm}$. Using the element-specific XMCD technique, we further found that the reduced spin moments arise from the Mn rather than the Co. Although the growth and magnetic properties of Heusler alloys have been explored for the last ten years, this study suggests that more work is required to improve the growth of Heusler alloy/ semiconductor hybrid spintronics structures to have a clean interface in order to make use of this encouraging material system. It is still not clear why spin polarization is much lower than the expected $100 \%$ and the predicted large magnetic moment transfer from the magnetic elements, such as Co, to other elements, such as Mn, is not observed in the ultrathin films of a few nanometers thick. Considering the large spin polarization, high Curie temperatures, fast switching, and the moderate barriers with either a Schottky contact or $\mathrm{MgO}$ layer, the half- 
metallic oxide/semiconductor hybrid structures might be one of the most promising second-generation spintronics systems. We would also like to point out that while the hybrid spintronic structures integrating half-metallic magnetic oxides and Heusler alloys are exciting for second-generation spintronics, a major challenge remains, however, to improve the Heusler alloy/semiconductor interface and to integrate the magnetic oxides with semiconductor devices to fulfill the promised great potential of these half metallic materials with a predicted spin polarization of $100 \%$.

\section{REFERENCES}

[1] Y. B. Xu and S. M. Thompson, Spintronic Materials and Technology. New York: Taylor \& Francis, 2006.

[2] H. Ohno, A. Shen, F. Matsukura, A. Oiwa, A. Endo, S. Katsumoto, and Y. Iye, "(Ga, Mn)As: A new diluted magnetic semiconductor based on GaAs," Appl. Phys. Lett., vol. 69, no. 3, pp. 363-365, 1996.

[3] K. W. Edmonds, K. Y. Wang, R. P. Campion, A. C. Neumann, N. R. S. Farley, B. L. Gallagher, and C. T. Foxon, "High-Curie-temperature Ga1-xMnxAs obtained by resistance-monitored annealing," Appl. Phys. Lett., vol. 81, no. 26, pp. 4991-4993, 2002.

[4] S. A. Chambers and R. F. C. Farrow, "New Possibilities for Ferromagnetic Semiconductors," MRS Bull., vol. 28, no. 10, pp. 729-734, 2003.

[5] X. Jiang, R. Wang, R. M. Shelby, R. M. Macfarlane, S. R. Bank, J. S. Harris, and S. S. P. Parkin, "Highly spin-polarized room-temperature tunnel injector for semiconductor spintronics using $\mathrm{MgO}(100)$," Phys. Rev. Lett., vol. 94, no. 5, p. 056601, 2005.

[6] G. A. Prinz, G. T. Rado, and J. J. Krebs, "Magnetic properties of singlecrystal $\{110\}$ iron films grown on GaAs by molecular beam epitaxy," J. Appl. Phys., vol. 53, no. 3, pp. 2087-2092, 1982.

[7] Y. B. Xu, E. T. M. Kernohan, D. J. Freeland, A. Ercole, M. Tselepi, and J. A. C. Bland, "Evolution of the ferromagnetic phase of ultrathin Fe films grown on GaAs(100)-4 × 6," Phys. Rev. B, vol. 58, no. 2, pp. 890-896, 1998.

[8] M. Brockmann, M. Zolfl, S. Miethaner, and G. Bayreuther, "In-plane volume and interface magnetic anisotropies in epitaxial Fe films on GaAs(0 0 1)," J. Magn. Magn. Mater., vol. 198, pp. 384-386, 1999.

[9] F. C. Voogt, T. T. M. Palstra, L. Niesen, O. C. Rogojanu, M. A. James, and T. Hibma, "Superparamagnetic behavior of structural domains in epitaxial ultrathin magnetite films," Phys. Rev. B, vol. 57, no. 14, pp. R8107-R8110, 1998.

[10] D. T. Margulies, F. T. Parker, F. E. Spada, R. S. Goldman, J. Li, R. Sinclair, and A. E. Berkowitz, "Anomalous moment and anisotropy behavior in Fe3O4 films," Phys. Rev. B, vol. 53, no. 14, pp. 9175-9187, 1996.

[11] C. Ruby, J. Fusy, and J.-M. R. Genin, "Preparation and characterisation of iron oxide films deposited on $\mathrm{MgO}(100)$," Thin Solid Films, vol. 352, no. $1-2$, pp. 22-28, 1999.

[12] W. Weiss and M. Ritter, "Stranski-Krastanov growth for Metal oxide heteroepitaxy: Iron oxides on Pt(111)," Phys. Rev. B, vol. 59, no. 7, pp. 5201-5213, 1999.

[13] R. A. de Groot, F. M. Mueller, P. G. van Engen, and K. H. J. Buschow, "New Class of Materials: Half-Metallic Ferromagnets," Phys. Rev. Lett., vol. 50, no. 25, pp. 2024-2027, 1983.

[14] Y. X. Lu, J. S. Claydon, Y. B. Xu, S. M. Thompson, K. Wilson, and G. van der Laan, "Epitaxial growth and magnetic properties of halfmetallic $\mathrm{Fe}_{3} \mathrm{O}_{4}$ on GaAs(100)," Phys. Rev. B, vol. 70, p. 233304, 2004.

[15] W. Van Roy, G. Borghs, and J. De Boeck, "Epitaxial growth of the half-metallic ferromagnet NiMnSb on $\operatorname{GaAs}(001)$, ," J. Crystal Growth, vol. 227-228, pp. 862-866, 2001.

[16] E. Girgis, P. Bach, C. Ruster, C. Gould, G. Schmidt, and L. Molenkamp, "Giant magnetoresistance in an epitaxial $\mathrm{NiMnSb} / \mathrm{Cu}$ / CoFe multilayer,", Appl. Phys. Lett., vol. 86, no. 14, pp. 142503-1-3-, 2005.

[17] J. Dong, L. Chen, J. Xie, T. Muller, D. Carr, C. Palmstrom, S. McKernan, Q. Pan, and R. James, "Epitaxial growth of ferromagnetic Ni2MnGa on GaAs(001) using NiGa interlayers,", J. Appl. Phys., vol. 88 , no. 12 , pp. 7357-7359, 2000.

[18] X. Dong, C. Adelmann, J. Xie, C. Palmstrom, X. Lou, J. Strand, P. Crowell, J.-P. Barnes, and A. Petford-Long, "Spin injection from the Heusler alloy Co2MnGe into A10.1Ga0.9As/GaAs heterostructures," Appl. Phys. Lett., vol. 86, no. 10, pp. 102107-1-102107-3, 2005.
[19] L. J. Singh, Z. H. Barber, Y. Miyoshi, W. R. Branford, and L. F. Cohen, "Structural and transport studies of stoichiometric and off-stoichiometric thin films of the full Heusler alloy Co2MnSi," J. Appl. Phys., vol. 95, no. 11, pp. 7231-7233, 2004.

[20] L. J. Singh, Z. H. Barber, A. Kohn, A. K. Petford-Long, Y. Miyoshi, Y. Bugoslavsky, and L. F. Cohen, "Interface effects in highly oriented films of the Heusler alloy Co2MnSi on GaAs(001)," J. Appl. Phys., vol. 99, no. 1, p. 013904, 2006.

[21] B. T. Thole, P. Carra, F. Sette, and G. van der Laan, "X-ray circular dichroism as a probe of orbital magnetization," Phys. Rev. Lett., vol. 68 , no. 12 , pp. $1943-1946,1992$

[22] C. T. Chen, Y. U. Idzerda, H.-J. Lin, N. V. Smith, G. Meigs, E. Chaban, G. H. Ho, E. Pellegrin, and F. Sette, "Experimental Confirmation of the X-Ray Magnetic Circular Dichroism Sum Rules for Iron and Cobalt," Phys. Rev. Lett., vol. 75, no. 1, pp. 152-155, 1995.

[23] E. Beaurepaire, H. Bulou, F. Scheurer, and J.-P. Kappler, "Magnetism and Synchrotron Radiation, Mittelwihr, 1996," Les Ditions Phys. Les Ulis, 1997.

[24] C. T. Chen, Y. U. Idzerda, H. J. Lin, N. V. Smith, G. Meigs, E. Chaban, G. H. Ho, E. Pellegrin, and F. Sette, "Experimental Confirmation of the X-Ray Magnetic Circular Dichroism Sum Rules for Iron and Cobalt," Phys. Rev. Lett., vol. 75, no. 1, pp. 152-155, 1995.

[25] C. D. Damsgaard, "Spin injection from epitaxial heusler alloy thin films into InGaAs/GaAs quantum wells," Ph.D. dissertation, Tech. Univ. Denmark, Lyngby, Denmark, 2006.

[26] S. C. Erwin, S.-H. Lee, and M. Scheffler, "Surface physics low-dimensional systems, and related topics-First-principles study of nucleation, growth, and interface structure of Fe/GaAs (10 pages)," Phys. Rev. B 65, vol. 205, p. 422, 2002.

[27] S. Mirbt, B. Sanyal, C. Isheden, and B. Johansson, "Surface physics, low-dimensional systems, and related topics-First-principles calculations of Fe on GaAs(100) (9 pages)," Phys. Rev. B 67, vol. 155, p. 421, 2003.

[28] P. J. Webster, "Magnetic and chemical order in heusler alloys containing cobalt and manganese," J. Phys. Chem. Solids, vol. 32, pp. 1221-1231, 1971.

[29] M. C. Hickey, C. D. Damsgaard, I. Farrer, S. N. Holmes, A. Husmann, J. B. Hansen, C. S. Jacobsen, D. A. Ritchie, R. F. Lee, G. A. C. Jones, and M. Pepper, "Spin injection between epitaxial Co2.4Mn1.6Ga and an InGaAs quantum well," Appl. Phys. Lett., vol. 86, no. 25, p. 252106, 2005.

[30] Y. Dong, C. Adelman, J. Q. Xie, C. J. Palmstrom, X. Lou, J. Strand, P. A. Cowell, J. P. Barnes, and A. K. Petford-Long, "Spin injection from the Heusler alloy Co2MnGe into Al0.1Ga0.9 As/GaAs heterostructures," Appl. Phys. Lett., vol. 86, no. 10, p. 102107, 2005.

[31] M. C. Hickey, S. N. Holmes, I. Farrer, D. A. Ritchie, M. Pepper, C. D. Damsgaard, C. S. Jacobsen, and J. B. Hansen, Appl. Phys. Lett., 2007, Submitted to

[32] W. Hiebert, A. Stankiewicz, and M. R. Freeman, "Activation of shallow boron acceptor in C/B coimplanted silicon carbide: A theoretical study," Phys. Rev. Lett., vol. 79, no. 6, pp. 1134-1137, 1997.

[33] J. Wu, N. D. Hughes, J. R. Moore, and R. J. Hicken, "Excitation and damping of spin excitations in ferromagnetic thin films," J. Magn. Magn. Mater., vol. 241, no. 1, pp. 96-109, 2002.

[34] C. Bunce, J. Wu, R. Chantrell, Y. X. Lu, and Y. B. Xu, "Picosecond magnetization dynamics of single-crystal $\mathrm{Fe} 3 \mathrm{O} 4$ thin films," IEEE Trans. Magn., 2008, submitted for publication.

[35] W. Eerenstein, T. T. M. Palstra, S. S. Saxena, and T. Hibma, "Spinpolarized transport across sharp antiferromagnetic boundaries," Phys. Rev. Lett., vol. 88, no. 10, p. 247204, 2002.

[36] S. M. Watts, C. Boothman, S. van Dijken, and J. M. D. Coey, "Magnetite Schottky barriers on GaAs substrates," Appl. Phys. Lett., vol. 86, no. 21 , p. $212108,2005$.

[37] J. G. Simmons, "Generalized formula for the electric tunnel effect between similar electrodes separated by a thin insulating film," J. Appl. Phys., vol. 34, no. 6, pp. 1793-1803, 1963.

[38] T. Kiyomura, Y. Maruo, and M. Gomi, "Electrical properties of $\mathrm{MgO}$ insulating layers in spin-dependent tunneling junctions using $\mathrm{Fe} 3 \mathrm{O} 4$," J. Appl. Phys., vol. 88, no. 8, pp. 4768-4772, 2000.

[39] S. Mitani, T. Moriyama, and K. Takanashi, " $\mathrm{Fe} / \mathrm{MgO} / \mathrm{FeCo}(100)$ epitaxial magnetic tunnel junctions prepared by using in situ plasma oxidation," J. Appl. Phys., vol. 93, no. 10, pp. 8041-8043, 2003.

Manuscript received March 03, 2008. Current version published December 17, 2008. Corresponding author: Y. B. Xu (e-mail: yx2@ohm.york.ac.uk). 